\title{
An experimental framework for quantifying the degree of intraguild predation in a three-species omnivorous food web in the field
}

Gen-Chang Hsu ( $\nabla$ genchanghsu@gmail.com )

National Taiwan University Department of Life Science https://orcid.org/0000-0002-6607-4382

\section{Research Article}

Keywords: feeding trial, food web, generalist predator, intraguild predation, mesopredator, omnivory, stable isotope analysis

Posted Date: June 6th, 2022

DOI: https://doi.org/10.21203/rs.3.rs-1268955/v2

License: (c) (i) This work is licensed under a Creative Commons Attribution 4.0 International License.

Read Full License 
1 Title

2 An experimental framework for quantifying the degree of intraguild predation in a three-

3 species omnivorous food web in the field

4

5 Author name and affiliation

6 Gen-Chang Hsu ${ }^{\text {a }}$

$7 \quad{ }^{a}$ Department of Life Science, National Taiwan University, Taipei, Taiwan

$8 \quad{ }^{a}$ No.1, Sec. 4, Roosevelt Rd., Taipei 10617, Taiwan (R.O.C.)

9

10 Corresponding author

11 Name: Gen-Chang Hsu

12 Address: No.1, Sec. 4, Roosevelt Rd., Taipei 10617, Taiwan (R.O.C.)

13 Email: genchanghsu@gmail.com

14 ORCID: https://orcid.org/0000-0002-6607-4382

15 
Abstract

17 Intraguild predation (IGP) is common in natural and human-managed systems and plays a critical role in food web dynamics. Although studies have documented the occurrence of IGP across a wide range of predator taxa, quantitative understanding regarding the degree/intensity of IGP remains lacking. I propose an experimental framework combining controlled feeding trials and stable isotope analysis to quantify the degree of IGP in a threespecies omnivorous food web (top predator + mesopredator + shared prey) in the field. The degree of IGP is defined as the proportion (in number) of mesopredator consumed in the total diet (shared prey + mesopredator) of the top predator. Feeding trials along with stable isotope analysis are used to construct a standard curve of the relationship between top predator's diet and shift in its nitrogen isotope signatures. The nitrogen isotope signatures of field-sampled top predator individuals are then analyzed and interpolated to the curve to estimate the degree of IGP in the field. The proposed framework leverages the strengths of different experimental approaches to studying trophic interactions, providing a tool for quantifying IGP in a more accurate (controlled feeding trials and standard IGP curve) and realistic (stable isotope analysis of field samples) fashion. The current framework can be further extended to food webs involving more complex interactions (e.g., cannibalism and multiple shared prey) and complemented with other approaches (e.g., molecular gut content analysis) to capture a more complete picture of IGP dynamics in the field.

\section{Keywords} feeding trial, food web, generalist predator, intraguild predation, mesopredator, omnivory, stable isotope analysis 
40 Intraguild predation (IGP) is common in natural and human-managed ecosystems (Polis \& Holt, 1992; Müller \& Brodeur, 2002; Arim \& Marquet, 2004) and is documented across a wide range of predator taxa (Polis et al., 1989; Gagnon et al., 2011). IGP could substantially affect the abundance and distribution of interacting species, generating ecological and evolutionary consequences for food web dynamics (Polis et al., 1989).

Previous studies have recorded the occurrence of IGP among arthropod predators through field observations of diet compositions (e.g., Nyffeler \& Sunderland, 2003; Birkhofer \& Wolters, 2012). Manipulative experiments (e.g., field cages) have also been used to assess the intensity of IGP (e.g., Denno et al., 2004) and could reveal the mechanisms underlying predator-prey interactions, allowing for strong causal inferences about IGP. Nonetheless, the confined settings in these experiments may potentially alter the encounter rates between organisms, thus leading to biased results (Uiterwaal et al., 2019).

Stable isotopes, particularly nitrogen isotope signatures $\left(\delta^{15} \mathrm{~N}\right)$, have been used to estimate the trophic levels of predators for inferences about IGP (e.g., Wise et al., 2006). It is suggested that IGP would increase the $\delta^{15} \mathrm{~N}$ of predators (Ponsard \& Arditi, 2000). For example, Rickers et al. (2006) conducted feeding experiments on wolf spiders (Alopecosa cuneata) and found a higher $\delta^{15} \mathrm{~N}$ of these top predators in IGP treatment. However, the study did not quantify the degree of IGP as the IGP treatment was binary (absence vs. presence of mesopredator) with constant numbers of shared prey and mesopredator. On top of that, the trophic levels of top predators in previous studies were often calculated based on assumed trophic discrimination factors (TDFs) (Svanbäck et al., 2015). Since TDFs are quite taxonspecific (Caut et al., 2009), this could lead to incorrect trophic level estimates and inferences about IGP in the field. 
Recently, researchers have applied molecular gut content analysis and immunological techniques to reliably detect the presence of certain food items in predators' diet (Gagnon et al., 2011), allowing for calculating the incidence rates of IGP (i.e., the percentage of top predator individuals with mesopredator detected in the gut contents). Nonetheless, the incidence rates may not necessarily reflect the degree of IGP in the system (Raso et al., 2014). For example, it is possible that a high percentage of top predator individuals feed on mesopredator yet each of them consumes on average a low proportion of mesopredator in the diet. In this case, a high incidence rate of IGP only provides an incomplete picture of IGP dynamics.

Quantifying IGP is a critical step towards a deeper understanding of food web dynamics. Research has attempted to predict the intensity/degree of IGP based on allometric theory (Schneider et al., 2012), yet empirical evidence remains scarce. To address this gap, I propose an experimental framework combining controlled feeding trials and stable isotope analysis of field samples to estimate the degree of IGP in a three-species omnivorous food web (top predator + mesopredator + shared prey). The degree of IGP is defined herein as the proportion (in number) of mesopredator consumed in the total diet (mesopredator + shared prey) of top predator. The feeding trials will experimentally link different levels of mesopredator consumption by top predator to the changes in top predator's nitrogen isotope signatures via a IGP standard curve, to which the nitrogen isotope signatures of fieldcollected top predator individuals are interpolated to estimate the degree of IGP in the field.

\section{The proposed experimental framework}

The proposed experimental framework consists of three main stages: (1) first feeding trial for stable isotope calibration of focal organisms, (2) second feeding trial for construction of 
standard IGP curve, and (3) collection of field samples for IGP estimation. I will illustrate the framework using an example of a terrestrial arthropod food web involving a spider top predator, a spider mesopredator, and a planthopper shared prey (Fig. 1a) in the following paragraphs.

The first feeding trial is to calibrate the nitrogen isotope signatures of the focal organisms. In this trial, the top predator and mesopredator are fed the shared prey for a period of time (Fig. 1b). The actual duration of feeding may vary depending on the species. For arthropods, a period of 5-10 days will allow predators to incorporate isotope signatures into their tissues and reach an isotopic equilibrium state with the shared prey (Quinby et al., 2020).

The second feeding trial is to simulate a full range of omnivory that the top predator may exhibit in the field for constructing a standard IGP curve. In this trial, the top predator is fed mixed diets with different proportions of shared prey and mesopredator individuals from the first feeding trial: (1) $100 \%$ shared prey, (2) $75 \%$ shared prey $+25 \%$ mesopredator, (3) $50 \%$ shared prey $+50 \%$ mesopredator, (4) $25 \%$ shared prey $+75 \%$ mesopredator, and (5) 100\% mesopredator (Fig. 1c). The actual numbers of shared prey and mesopredator supplied can be determined based on their field densities. To avoid the potential interfering effects of mesopredator feeding on the shared prey, the prey items are presented to the top predator one at a time in a randomized sequence instead of all at once. This also allows the researcher to ensure that a prey item is consumed by the top predator before the next item is presented.

At the end of the second trial (same duration as the first feeding trial to allow for the incorporation of prey isotope signatures into predator's tissues), the top predator individuals in each diet treatment as well as the shared prey are prepared for stable isotope analysis to obtain their $\delta^{15} \mathrm{~N}$ values. The difference in $\delta^{15} \mathrm{~N}$ between the top predator and the shared prey (baseline) is computed $\left(\delta^{15} \mathrm{~N}_{\text {predator }}-\delta^{15} \mathrm{~N}_{\text {prey }}\right.$; experimental $\left.\Delta^{15} \mathrm{~N}\right)$, and a standard curve is 
constructed by fitting a non-linear regression on the experimental $\Delta^{15} \mathrm{~N}$ against the proportion of mesopredator in the diet (Fig. 1d).

Finally, field samples of top predator and shared prey are collected for stable isotope analysis. The shared prey individuals are pooled to obtain a single baseline $\delta^{15} \mathrm{~N}$, while the $\delta^{15} \mathrm{~N}$ of top predator individuals are analyzed separately and thus each predator has its own empirical $\Delta^{15} \mathrm{~N}$. The degree of IGP at the individual level can then be estimated by interpolating the individual empirical $\Delta^{15} \mathrm{~N}$ to the standard IGP curve (Fig. 1e). The mean and standard error of these individual IGP estimates can provide a measure of the average degree of IGP in the field and the uncertainty around the mean estimate at the population level.

A hypothetical example of standard IGP curve construction and estimation of IGP with field samples is shown in Fig. 2. In this example, each diet treatment contains five top predator individuals, each of which is fed 12 prey items during the feeding period. After the feeding trial, the experimental $\Delta^{15} \mathrm{~N}$ of these predator individuals are determined and used to construct a standard IGP curve (Fig. 2a). 20 top predator and 30 shared prey individuals (pooled) are then collected from the field for stable isotope analysis and determination of empirical $\Delta^{15} \mathrm{~N}$. An IGP estimate is calculated for each top predator individual so that there will be a total of 20 estimates, which are further averaged to quantify the degree of IGP at the population level (Fig. 2b).

\section{Applications}

The proposed framework leverages the strengths of different approaches to studying trophic interactions - the controlled feeding trials combined with stable isotope analysis can yield accurate experimental $\Delta^{15} \mathrm{~N}$ for constructing a standard IGP curve, whereas the 
empirical $\Delta^{15} \mathrm{~N}$ derived from field samples reflects the trophic interactions under natural settings. Additionally, the framework is robust to variations in background isotope signatures because the IGP estimation is based on the difference in nitrogen isotope signatures $\left(\Delta^{15} \mathrm{~N}\right)$ rather than the original values $\left(\delta^{15} \mathrm{~N}\right)$, thus allowing for comparisons across sites or systems with distinct background isotope signatures.

The framework can be implemented along environmental gradients or under different field treatments to investigate how various abiotic and biotic factors affect IGP interactions. For instance, one can quantify and compare the degree of IGP across altitudes to examine whether omnivory changes with temperature, precipitation, or vegetation. Moreover, this study uses a spider as an example of top predator, but the framework applies to other generalist predators as well, provided that they are amenable to feeding trials and easy to collect in the field.

The systems ideal for implementing the proposed framework are those with clear IGP patterns and relatively simple trophic interaction networks, which can minimize the potential interfering effects of non-focal species on the IGP interactions among focal organisms (Vance-Chalcraft et al., 2007). One of such systems is agricultural system, in which IGP occurs frequently (Polis et al., 1989; Rosenheim et al., 1995) and the food webs are generally less complex compared with most natural ecosystems. Furthermore, understanding the degree of IGP in agricultural field can have useful implications for practitioners, for example, evaluation of the effectiveness of biocontrol agents in pest control programs (Müller \& Brodeur, 2002).

A potential limitation of the proposed framework is that multiple mesopredators and shared prey with different isotope signatures may exist in the field, which could introduce variations into the IGP estimates for individual top predators. Yet, given sufficiently large 
158 field samples, the average of these individual estimates should fairly reflect the overall IGP 159 patterns in the field at the population level. Therefore, although the isotope signatures of top 160 predators could represent multiple dietary items over time and space, the framework still 161 provides a tool for assessing IGP in a more quantitative and realistic fashion. predator-predator-prey trophic interactions and help predict the community structure and

\section{Acknowledgements}

I thank William J.-A. Ou and Po-Ju Ke for the useful comments on this manuscript.

\section{Conflict of interest}

The author declares no conflict of interest regarding this manuscript.

\section{Data availability statement}

Data sharing is not applicable to this manuscript as no new data were generated or analyzed. 


\section{Reference}

Arim, M. \& Marquet, P.A. (2004) Intraguild predation: a widespread interaction related to species biology. Ecology Letters, 7, 557-564.

Birkhofer, K. \& Wolters, V. (2012) The global relationship between climate, net primary production and the diet of spiders. pp. 100-108. Wiley Online Library.

Caut, S., Angulo, E. \& Courchamp, F. (2009) Variation in discrimination factors $(\Delta 15 \mathrm{~N}$ and $\Delta 13 \mathrm{C})$ : the effect of diet isotopic values and applications for diet reconstruction. Journal of Applied Ecology, 46, 443-453.

Denno, R.F., Mitter, M.S., Langellotto, G.A., Gratton, C. \& Finke, D.L. (2004) Interactions between a hunting spider and a web-builder: consequences of intraguild predation and cannibalism for prey suppression. Ecological entomology, 29, 566-577.

Gagnon, A.-È., Heimpel, G.E. \& Brodeur, J. (2011) The ubiquity of intraguild predation among predatory arthropods. PLoS One, 6, e28061.

Müller, C.B. \& Brodeur, J. (2002) Intraguild predation in biological control and conservation biology. Biological Control, 25, 216-223.

Nyffeler, M. \& Sunderland, K.D. (2003) Composition, abundance and pest control potential of spider communities in agroecosystems: a comparison of European and US studies. Agriculture, Ecosystems \& Environment, 95, 579-612.

Pahl, K.B., Yurkowski, D.J., Lees, K.J. \& Hussey, N.E. (2020) Measuring the occurrence and strength of intraguild predation in modern food webs. Food Webs, e00165.

Polis, G.A. \& Holt, R.D. (1992) Intraguild predation: the dynamics of complex trophic interactions. Trends in ecology \& evolution, 7, 151-154. 
Polis, G.A., Myers, C.A. \& Holt, R.D. (1989) The ecology and evolution of intraguild predation: potential competitors that eat each other. Annual Review of Ecology and Systematics, 20, 297-330.

Ponsard, S. \& Arditi, R. (2000) What can stable isotopes ( $\delta 15 \mathrm{~N}$ and $\delta 13 \mathrm{C})$ tell about the food web of soil macro-invertebrates? Ecology, 81, 852-864.

Quinby, B.M., Creighton, J.C. \& Flaherty, E.A. (2020) Stable isotope ecology in insects: a review. Ecological Entomology, 45, 1231-1246.

Raso, L., Sint, D., Mayer, R., Plangg, S., Recheis, T., Brunner, S., Kaufmann, R. \& Traugott, M. (2014) Intraguild predation in pioneer predator communities of alpine glacier forelands. Molecular ecology, 23, 3744-3754.

Rickers, S., Langel, R. \& Scheu, S. (2006) Stable isotope analyses document intraguild predation in wolf spiders (Araneae: Lycosidae) and underline beneficial effects of alternative prey and microhabitat structure on intraguild prey survival. Oikos, 114, $471-478$.

Rosenheim, J.A., Kaya, H.K., Ehler, L.E., Marois, J.J. \& Jaffee, B.A. (1995) Intraguild predation among biological-control agents: theory and evidence. Biological Control, 5, 303-335.

Schneider, F.D., Scheu, S. \& Brose, U. (2012) Body mass constraints on feeding rates determine the consequences of predator loss. Ecology Letters, 15, 436-443.

Svanbäck, R., Quevedo, M., Olsson, J. \& Eklöv, P. (2015) Individuals in food webs: the relationships between trophic position, omnivory and among-individual diet variation. Oecologia, 178, 103-114.

Uiterwaal, S.F., Dell, A.I. \& DeLong, J.P. (2019) Arena size modulates functional responses via behavioral mechanisms. Behavioral Ecology, 30, 483-489. 
226 Vance-Chalcraft, H.D., Rosenheim, J.A., Vonesh, J.R., Osenberg, C.W. \& Sih, A. (2007) The influence of intraguild predation on prey suppression and prey release: a meta-analysis. Ecology, 88, 2689-2696.

229 Wise, D.H., Moldenhauer, D.M. \& Halaj, J. (2006) Using stable isotopes to reveal shifts in prey consumption by generalist predators. Ecological Applications, 16, 865-876. 
233 Figure 1.

(a) Top predator קर्व

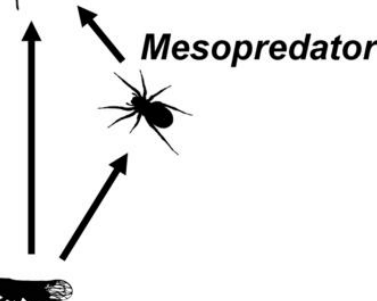

살

Shared prey (b)

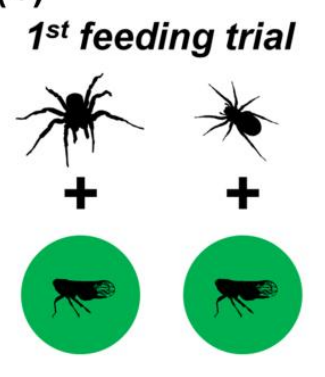

(e) (c)
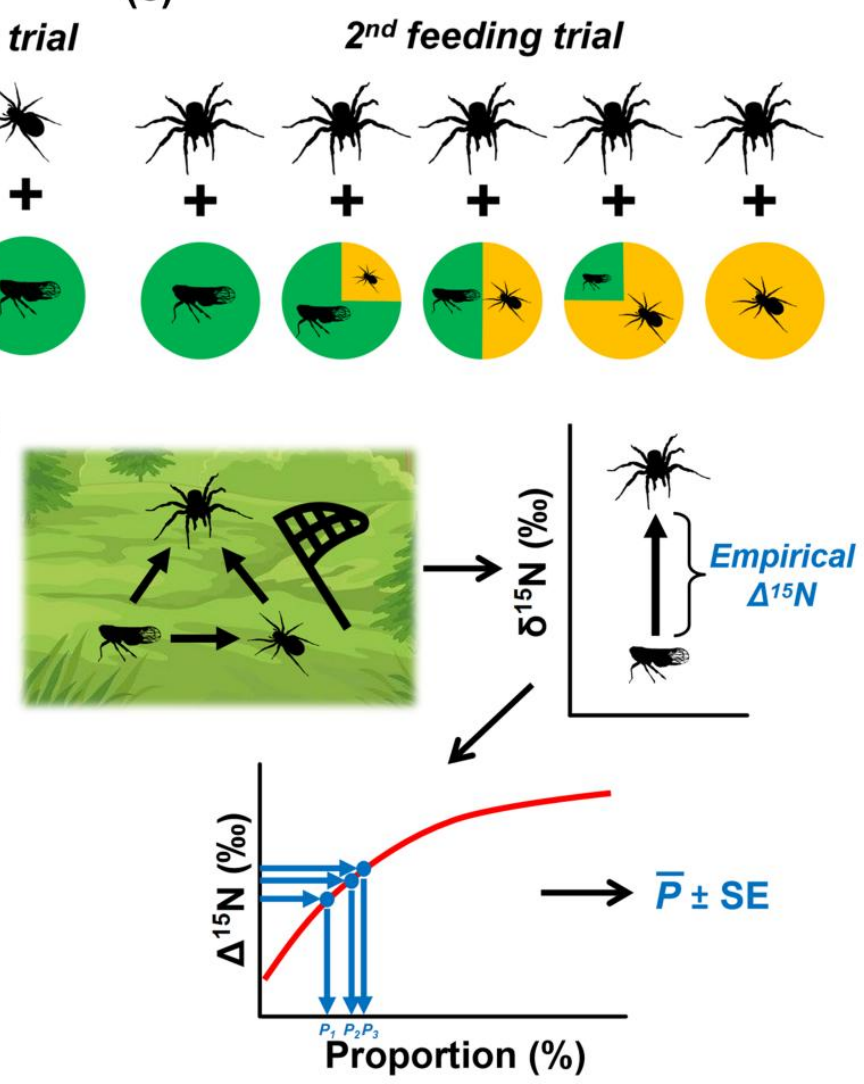

) (d)

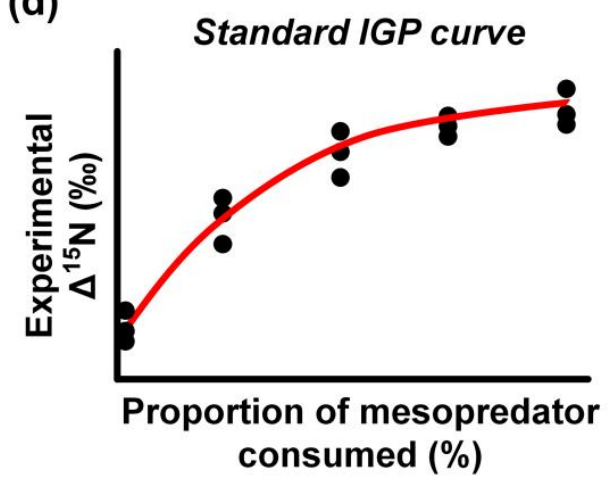

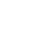

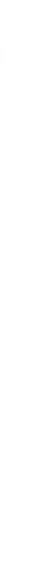

Figure 1. A schematic diagram of the proposed experimental framework for quantifying the

236 degree of intraguild predation (IGP) in a three-species omnivorous food web involving a spider

237 top predator, a spider mesopredator, and a planthopper shared prey. See "The proposed 238 experimental framework" for detailed descriptions of the steps in the framework. 
(a)

\begin{tabular}{|c|c|c|c|c|c|}
\hline $\begin{array}{c}\text { Diet } \\
\text { treatment }\end{array}$ & - & $*$ & $*$ & $*$ & $*$ \\
\hline $\mathbf{N}$ & $12 / 0$ & $9 / 3$ & $6 / 6$ & $3 / 9$ & $0 / 12$ \\
\hline $\boldsymbol{P}$ & 0.00 & 0.25 & 0.50 & 0.75 & 1.00 \\
\hline & 1.0 & 1.4 & 2.2 & 2.4 & 2.9 \\
& 0.8 & 1.8 & 2.1 & 2.4 & 2.7 \\
$\boldsymbol{\Delta}^{\mathbf{1 5}} \mathbf{N}(\mathbf{\% 0})$ & 0.9 & 1.3 & 1.7 & 2.3 & 2.7 \\
& 0.9 & 1.5 & 2.2 & 2.5 & 2.6 \\
& 1.0 & 1.5 & 2.0 & 2.3 & 2.8 \\
\hline
\end{tabular}

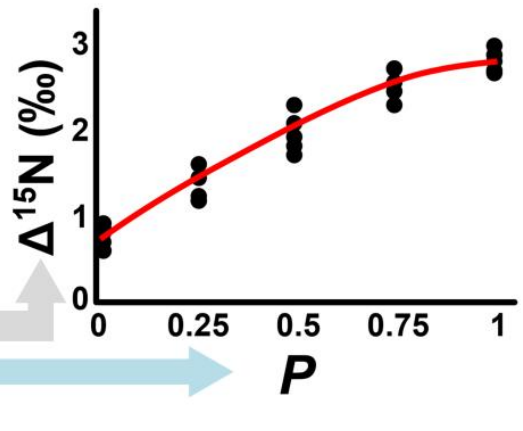

(b)
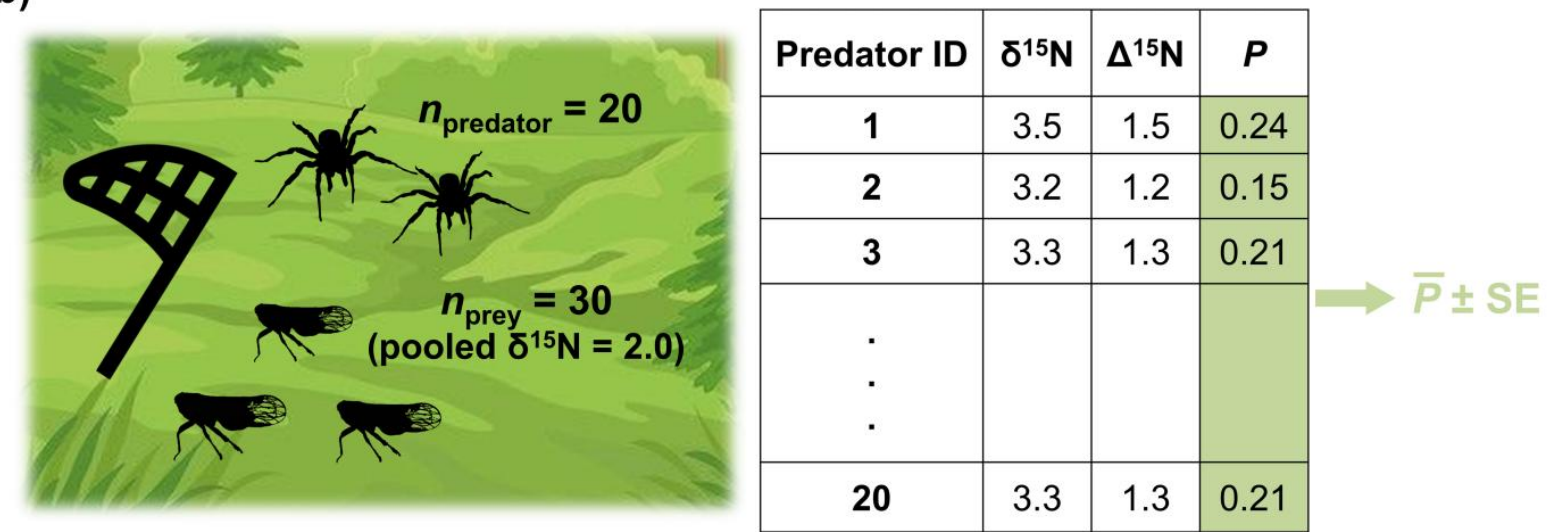

242 Figure 2. A hypothetical example of (a) standard IGP curve construction using data collected

243 from the second feeding trial and (b) estimation of IGP with field samples of top predator and

244 shared prey. $N$ : number of shared prey/mesopredator in the mixed diet; $P$ : proportion of 245 mesopredator consumed (\%). 